new model to existing models of model for end-stage liver disease (MELD), Child-Pugh scores and Glasgow Blatchford Score.

Results The 6-week overall mortality rate was $12.3 \%$. Multivariate analysis showed that Mean Arterial Pressure (MAP), model for end-stage liver disease (MELD), high-risk stigmata of esophageal varices or peptic ulcer on endoscopic finding and the Glasgow Blatchford Score were independent predictors of mortality. A new logistic model using these variables was developed. This model's AUROC was 0.934, which was significantly higher than that of MELD (0.721), MAP (0.842), and Glasgow Blatchford Score (0.904). Two external validation studies showed that the AUROC of our model was consistently higher than 0.954 . (figure 1)

Conclusions Our new simplified model accurately and consistently predicted 6-week mortality in patients with acute variceal bleeding using objective variables measured at admission. Our system can be used to identify high-risk acute variceal bleeding patients.

\section{IDDF2019-ABS-0079 IMPACT OF TIME-TO-SURGERY ON THE PROGNOSIS OF HEPATOCELLULAR CARCINOMA PATIENTS AT BCLC STAGE 0- A AFTER LIVER RESECTION}

Mengchao Wei* ${ }^{*}$, Shuling Chen, Zhenwei Peng, Zebin Chen, Han Xiao, Ming Kuang. The First Affiliated Hospital of Sun Yat-sen University, China

\subsection{6/gutjnl-2019-IDDFabstracts. 12}

Background Long waiting time before surgery caused by preoperative interventions may lead to tumor progression and worse prognosis in patients with hepatocellular carcinoma (HCC). The impact of time-to-surgery (TTS) on the prognosis of HCC patients has not been well illustrated in Chinese HCC patients. We tried to clarify the TTS issue in this study in order to provide new perspectives for making rational treatment timing for surgery.

Methods We enrolled 1051 HCC patients at BCLC stage 0-A with primary liver resection from three centers in China. Patients were divided into two groups according to different cut-off values of TTS (14 days, 21 days, 28 days, 35 days, 42 days). The primary endpoints were recurrence-free survival (RFS) and overall survival (OS).

Results The median TTS of HCC patients at BCLC stage 0-A was 14 days. Patients in the longer TTS group had significantly longer RFS and OS than those in the shorter TTS group when cut off values of TTS were 28 (RFS: $P=0.044$; OS: $P=0.004$ ) and 35 (RFS: $P=0.016$; OS: $P=0.044$ ) days.
TTS did not produce a significant impact on patients' RFS and OS when cut off values were 14, 21 and 42 days.

Conclusions TTS between 28 and 42 days may be appropriate for Chinese HCC patients at BCLC stage 0-A to receive surgery.

\section{IDDF2019-ABS-0108 HEPATIC DECOMPENSATION RISK IS REDUCED, BUT NOT ELIMINATED AFTER DIRECT-ACTING ANTIVIRALS: THE ROLE OF SPLEEN STIFFNESS MEASUREMENT}

${ }^{1}$ Giovanni Marasco*, 'Elton Dajti, ${ }^{1}$ Federico Ravaioli, ${ }^{2}$ Antonio Colecchia, ${ }^{1}$ Maria Letizia Bacchi Reggiani, ${ }^{3}$ Agostino Colli, 'Luigina Vanessa Alemanni, 'Maria Rosa Tame, ${ }^{1}$ Pietro Andreone, ${ }^{1}$ Stefano Brillanti, ${ }^{1}$ Francesco Azzaroli, ${ }^{1}$ Giuseppe Mazzella, ${ }^{1}$ Davide Festi. ${ }^{1}$ University of Bologna, Italy; ${ }^{2}$ Borgo Trento Hospital Verona, Italy; ${ }^{3}$ A. Manzoni Hospital Lecco, Italy

\subsection{6/gutjnl-2019-IDDFabstracts. 13}

Background Little evidence is available on the risk of hepatic decompensation (HD), mainly those related to portal hypertension (PH), after direct-acting antivirals (DAAs) in patients with HCV-related advanced chronic liver disease (ACLD). Our aims were: a) to evaluate the incidence of HD after DAAs, as well as the effect of such treatments on HD development and b) to assess the role of liver (LSM) and spleen (SSM) stiffness measurement in HD prediction after sustained virologic response (SVR).

Methods We performed in our tertiary centre a cohort study in 146 ACLD patients treated with DAAs and with available LSM and SSM both before and 6 months after end-of-treatment (EOT). A historical cohort of 92 consecutively enrolled untreated cirrhotic patients with active HCV-infection was used as a control group. A propensity score stabilized inverse probability weighting approach was used to account for differences between groups. Time-dependent models for HD prediction after SVR were applied to account for changes in LSM and SSM after DAA therapy.

Results Median follow-up in the DAA cohort was 33.5 (22 38) months. The HD incidence in this cohort was 7.07 (4.56-10.96) per 100 person-years (PYs), significantly higher than in the active HCV cohort, 19.75 (13.81-28.25) per 100 PYs. DAA therapy was an independent protective factor for HD development (hazard ratio [HR], 0.177; 95\%Interval-ofconfidence [CI], 0.081-0.390) (figure 1A), whereas previous HD (HR, 5.982; 95\%CI 2.434-14.702) and higher SSM values (HR, 1.025; 95\%CI 1.006-1.045) were associated with a higher risk of the event. $S S M \geq 54 \mathrm{kPa}$ was independently associated with HD despite SVR achievement (HR, 4.678;
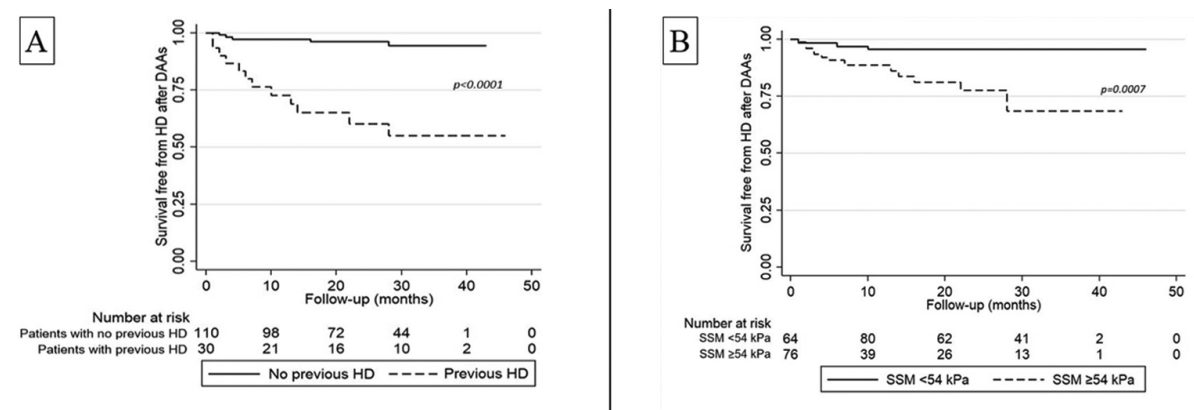
95\%IC 1.307-16.744) (figure 1B). The time-dependent model including SSM values at baseline and at 6 months after EOT predicted post-SVR HD development better than the models including LSM and its changes after therapy.

Conclusions The risk of $\mathrm{HD}$ is markedly reduced after DAA therapy, but not abolished. SSM is confirmed as an accurate surrogate of portal hypertension, able to stratify for the risk of HD development after DAA therapy more accurately than LSM.

\section{IDDF2019-ABS-0196 LONG TERM OUTCOMES OF UTILIZING DONATION AFTER CIRCULATORY DEATH GRAFTS IN LIVER TRANSPLANTATION - AN AUSTRALIAN 12-YEAR COHORT STUDY}

Vinay Sastry*, Keval Pandya, Mara Panlilio, Claire West, Susan Virtue, Mark Wells, Michael Crawford, Carlo Pulitano, Simone Strasser, Geoff McCaughan, Avik Majumdar, Ken Liu. Australian National Liver Transplant Unit, Royal Prince Alfred Hospital, Sydney, Australia

\subsection{6/gutjnl-2019-IDDFabstracts. 14}

Background Organ shortage for liver transplantation (LT) is a major problem worldwide. Use of donation circulatory death donors (DCD) has been one strategy to expand the donor pool; however, data on long term outcomes in DCD graft recipients are mixed. We studied the characteristics, utilization and recipient outcomes of DCDs.

Methods We retrospectively studied adults who underwent deceased LT between 2006-2018. Donor and recipient data at LT and recipient outcomes were collected from a prospective database. Criteria for DCD selection at our center include all of the following: donor age $<50$ years, BMI $<30 \mathrm{~kg} / \mathrm{m}^{2}$, and circulatory arrest within 30 minutes of treatment withdrawal in otherwise suitable liver donors. The primary outcome of interest was graft survival (time to retransplantation or death).

Results During the study period (median follow-up 50.6months), 739 donors were utilized for LT with $53(7.2 \%)$ being DCDs. Compared to donation after brain death donors (DBDs), DCDs were younger (30 vs. 50years), more likely to have history of predonation cardiac arrest $(71.2 \%$ vs. $34.8 \%)$, had longer intubation time (3 vs. 2days), less inotrope requirements $(32.7 \%$ vs. $7.8 \%$ on no agents) and higher AST (59 vs. 46U/L) (median values presented, all $P<0.01$ ). DCDs had shorter cold ischemia time $(5.75$ vs. 6.85 hours, $P=0.005)$ and

A

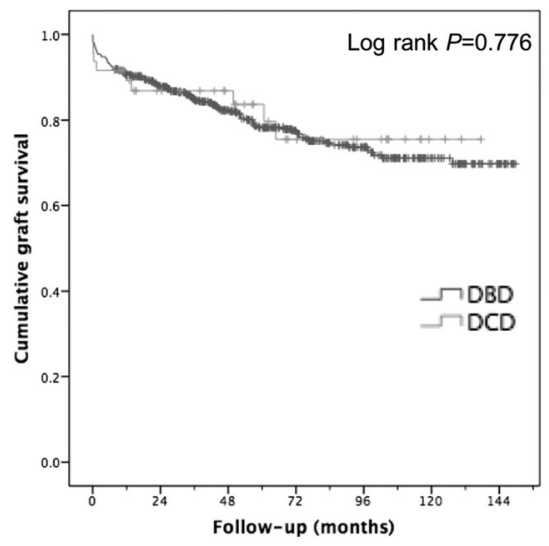

higher donor risk index (Feng et al. 2006) (1.68 vs. 1.56, $P<0.001)$. As per our unit policy, recipients of DCD grafts were less likely to be PSC $(1.9$ vs. 9.0\%, $P=0.076)$ or retransplant patients ( 0 vs. $6 \%, P=0.067$ ). Similarly, DCD recipients did not receive split grafts $(0$ vs. $15.3 \%, P<0.001)$. The proportion of DCDs among utilized grafts increased from $4.8 \%$ (2006-2009) to $7.8 \%$ (2010-2012) and remained stable afterward. 157 patients experienced graft loss during follow-up (31 retransplants, 126 deaths). DCD grafts had similar long term graft survival compared to DBDs, although DCDs recipients with high preLT MELD (>20) appeared to have worse outcomes (figure 1).

Conclusions Long term outcomes of DCD grafts are similar to DBD grafts especially when matched with appropriately selected recipients (first transplant, nonPSC patients with low MELD).

\section{IDDF2019-ABS-0332 COMPARISON OF ANTIBIOTICS FOR THE PRIMARY AND SECONDARY PROPHYLAXIS OF SPONTANEOUS BACTERIAL PERITONITIS: A SYSTEMATIC REVIEW AND NETWORK META-ANALYSIS WITH BAYESIAN APPROACH}

Hariom Soni* ${ }^{*}$ Praveen Kumar, Balaji Bellam, Shubhra Mishra, Dhruv Mahendru, Harshal Mandavdhare, Vishal Sharma. Postgraduate Institute of Medical Education and Research, India

\subsection{6/gutjnl-2019-IDDFabstracts. 15}

Background Spontaneous bacterial peritonitis is an infection of high serum-ascites albumin gradient (SAAG) ascites which usually occurs with cirrhosis. Various antibiotics are used for primary and secondary prevention of spontaneous bacterial peritonitis (SBP). We planned this study to systematically review the evidence from randomised studies comparing various antibiotics with placebo or one another for primary and secondary prevention of SBP.

Methods We did a comprehensive literature search using various databases (i.e. MEDLINE via Ovid and PubMed, Embase, Scopus, Web of science, LILIAC, Cochrane Central Register of Controlled Trials and clinicaltrials.gov from inception to $6^{\text {th }}$ September 2018. The search strategy contained no language restrictions and used following keywords 'Liver cirrhosis', 'Chronic liver disease', 'Chronic Liver Failure', 'Primary

B

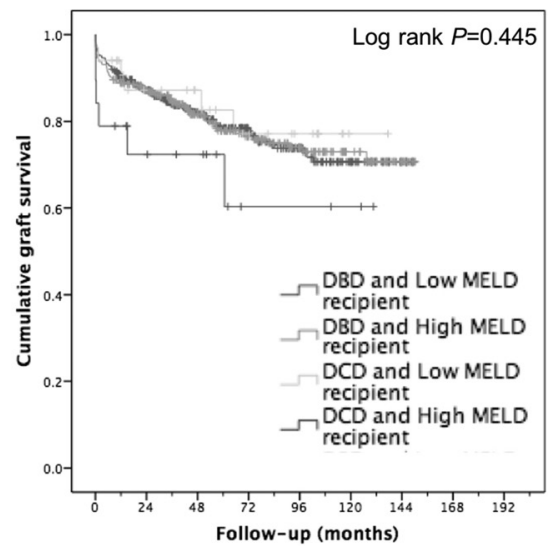

